\title{
GRS Method for Uncertainty and Sensitivity Evaluation of Code Results and Applications
}

\author{
Horst Glaeser \\ Gesellschaft für Anlagen- und Reaktorsicherheit (GRS) mbH, Forschungsinstitute, 85748 Garching, Germany \\ Correspondence should be addressed to Horst Glaeser, horst.glaeser@grs.de
}

Received 8 May 2007; Accepted 14 February 2008

Recommended by Cesare Frepoli

During the recent years, an increasing interest in computational reactor safety analysis is to replace the conservative evaluation model calculations by best estimate calculations supplemented by uncertainty analysis of the code results. The evaluation of the margin to acceptance criteria, for example, the maximum fuel rod clad temperature, should be based on the upper limit of the calculated uncertainty range. Uncertainty analysis is needed if useful conclusions are to be obtained from "best estimate" thermalhydraulic code calculations, otherwise single values of unknown accuracy would be presented for comparison with regulatory acceptance limits. Methods have been developed and presented to quantify the uncertainty of computer code results. The basic techniques proposed by GRS are presented together with applications to a large break loss of coolant accident on a reference reactor as well as on an experiment simulating containment behaviour.

Copyright (C) 2008 Horst Glaeser. This is an open access article distributed under the Creative Commons Attribution License, which permits unrestricted use, distribution, and reproduction in any medium, provided the original work is properly cited.

\section{INTRODUCTION}

Best estimate computer codes are used to calculate postulated loss of coolant accidents and transient in a realistic way and not in a conservative way. There is an increasing interest in computational reactor safety analysis to replace the conservative evaluation model calculations by best estimate calculations supplemented by a quantitative uncertainty analysis. The USA Code of Federal Regulation (CFR) 10 CFR 50.46 [1], for example, allows either to use a best estimate code plus identification and quantification of uncertainties, or the conservative option using conservative computer code models listed in Appendix K of the CFR, Title 10, Part 50.

Code predictions are uncertain due to several sources of uncertainty, like code models as well as uncertainties of plant and fuel parameters. These uncertainties, for example, come from scatter of measured values, approximations of modelling, variation and imprecise knowledge of initial and boundary conditions. Computer code models are developed based on experiments which can simulate the complex behaviour of a reactor plant under accident conditions in a simplified way only. Most of the experiments are performed in small scale compared to plant size. Uncertainty due to imprecise knowledge of parameter values in calculations is quantified by ranges and probability distributions. These distributions should be taken into account for input parameters instead of one discrete value only.

Stochastic variability due to possible component failures of the reactor plant is not considered in an uncertainty analysis. The single failure criterion is still taken into account in a deterministic way. This is a superior principle of safety analysis and requirements of redundance. The probability of system failures is part of probabilistic safety analyses, not of demonstrating the effectiveness of emergency core cooling systems.

The aim of the uncertainty analysis is at first to identify and quantify all potentially important uncertain parameters. Their propagation through computer code calculations provides probability distributions and ranges for the code results. The evaluation of the margin to acceptance criteria, for example, the maximum fuel rod clad temperature, should be based on the upper limit of this distribution for the calculated temperatures, see Figure 1. Uncertainty analysis is needed if useful conclusions with regard to prediction capability, such as maximum cladding temperature, are to be obtained from "best estimate" thermal-hydraulic code calculations, otherwise single values of unknown accuracy would be presented for comparison with limits for acceptance. 


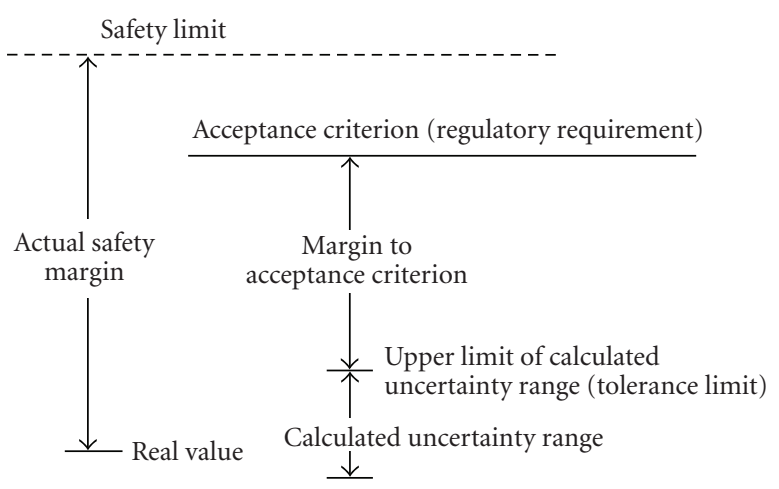

FIGURE 1: Margin illustration.

Section 2 describes the GRS method, Section 3 presents examples of application of the GRS method, and Section 4 provides conclusions.

\section{DESCRIPTION OF THE GRS METHOD}

Among others, GRS method [2] has been developed for the determination of uncertainties. The state of knowledge about all uncertain parameters is described by ranges and probability distributions, Figure 2. In order to get information about the uncertainty of computer code results, a number of code runs have to be performed. For each of these calculation runs, all identified uncertain parameters are varied simultaneously. Uncertain parameters are uncertain input values, models, initial and boundary conditions, numerical values like convergence criteria and maximum time step size, and so forth. Model uncertainties are expressed by adding on or multiplying correlations by corrective terms, or by a set of alternative model formulations. Uncertainties in noding, to describe the important phenomena, are to be taken into account in the code validation process. However, alternative noding schemes can be included in the uncertainty analysis. Code validation results are a fundamental basis to quantify parameter uncertainties.

The selection of parameter values according to their specified probability distributions, their combination, and the evaluation of the calculation results requires a method. Following a proposal by GRS, the central part of the method is a set of statistical techniques. The advantage of using these techniques is that the number of code calculations needed is independent of the number of uncertain parameters. In each code calculation, all uncertain parameters are varied simultaneously. In order to quantify the effect of these variations on the result, statistical tools are used. Because the number of calculations is independent of the number of uncertain parameters, no a priori ranking of input parameters is necessary to reduce their number in order to cut computation cost. The ranking is a result of the analysis as described later.

The number of code calculations depends on the requested probability content and confidence level of the statistical tolerance limits used in the uncertainty statements of
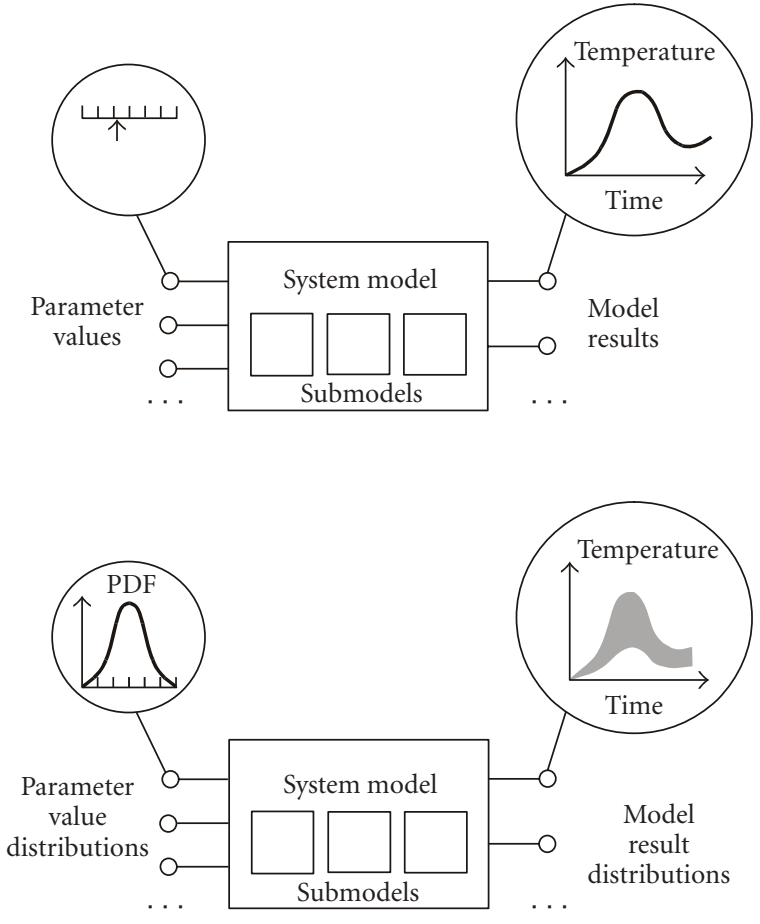

FIGURE 2: Consideration of input parameter value ranges instead of discrete values in the GRS method.

the results. The required minimum number $\mathrm{n}$ of these calculation runs is given by Wilks' formula $[3,4]$, for example, for one-sided tolerance limits: $1-a^{n} \geq b$, where $b \times 100$ is the confidence level (\%) that the maximum code result will not be exceeded with the probability $a \times 100$ (\%) (percentile) of the corresponding output distribution, which is to be compared to the acceptance criterion. The confidence level is specified to account for the possible influence of the sampling error due to the fact that the statements are obtained from a random sample of limited size. For two-sided statistical tolerance intervals, the formula is: $1-a^{n}-n(1-a) a^{n-1} \geq$ $b$. The minimum number of calculations can be found in Table 1 .

The probabilistic treatment of parameter uncertainties allows quantifying their state of knowledge. This means, in addition to the uncertainty range, the knowledge is expressed by probability density functions or probability distributions. This interpretation of probability is used for a parameter with a fixed but unknown or inaccurately known value. The classical interpretation of probability as the limit of a relative frequency, expressing the uncertainty due to stochastic variability, is not applicable here.

The probability distribution can express that some values in the uncertainty range are more likely to be the appropriate parameter value than others. In the case that no preferences can be justified, uniform distribution will be specified, that is, each value between minimum and maximum is equally likely to be the appropriate parameter value. As the consequence of this specification of probability distributions of input parameters, the computer code results also show a 
TABLE 1: Minimum number of calculations $n$ for one-sided and two-sided statistical tolerance limits.

\begin{tabular}{ccccccc}
\hline & \multicolumn{2}{c}{ One-sided statistical tolerance limits } & \multicolumn{3}{c}{ Two-sided statistical tolerance limits } \\
\hline$b / a$ & 0.90 & 0.95 & 0.99 & 0.90 & 0.95 & 0.99 \\
0.90 & 22 & 45 & 230 & 38 & 77 & 388 \\
0.95 & 29 & 59 & 299 & 46 & 93 & 473 \\
0.99 & 44 & 90 & 459 & 64 & 130 & 662 \\
\hline
\end{tabular}

probability distribution, from which uncertainty limits or intervals are derived.

A total number of $n$ code runs are performed varying simultaneously the values of all uncertain input parameters, according to their distribution. The $n$ values of the considered output parameters are ordered: $Y(1)<Y(2) \cdots<$ $Y(n-1)<Y(n)$. Therefore, the name-order statistics is used for Wilks' formula. On the basis of this ranking, the 95th percentile value with a confidence level of $95 \%$ is obtained by selecting $Y(n)$ with $n=59$ for the one-sided tolerance limit, for example. A 5th percentile value with a confidence level of $95 \%$ is obtained by selecting $Y(1)$ with $n=59$. A (95\%/95\%) two-sided tolerance limit is obtained by selecting $Y(1)$ and $Y(n)$ with $n=93$.

Another important feature of the method is that one can evaluate sensitivity measures of the importance of parameter uncertainties for the uncertainties of the results. These measures give a ranking of input parameters. This information provides guidance as to where to improve the state of knowledge in order to reduce the output uncertainties most effectively, or where to improve the modelling of the computer code. Sensitivity measures like standardised rank regression coefficients, rank correlation coefficients, and correlation ratios permit a ranking of uncertainties in model formulations, input data, and so forth, with respect to their relative contribution to code output uncertainty. The difference to other known uncertainty methods, for example, [5], is that the ranking is a result of the analysis and not of prior estimates and judgements. This prior setup of a phenomena identification and ranking table (PIRT) by extensive expert staffhours in [5] is known to be very costly. Uncertainty statements and sensitivity measures are available simultaneously for all single-valued (e.g., peak clad temperature) as well as continuous valued (time dependent) output quantities of interest. The method relies only on actual code calculations without using approximations like fitted response surfaces. Similar methods based on the GRS method, and an alternative uncertainty method is presented in [6].

The different steps of the uncertainty analysis according to the GRS method are supported by the software system for uncertainty and sensitivity analyses (SUSA) developed by GRS [7]. They provide a choice of statistical tools to be applied during the uncertainty and sensitivity analysis.

\section{APPLICATIONS}

The GRS method for uncertainty and sensitivity evaluation of code results can be used for different codes to investigate the combined influence of all potentially important uncer- tainties on the calculation results. Several applications have been performed in GRS to investigate loss of coolant from the primary and secondary coolant systems of pressurised water reactors, as well as related experiments. For these analyses, we used the thermal-hydraulic computer code ATHLET. Another uncertainty and sensitivity analysis was performed calculating an experiment simulating containment behaviour using the computer code COCOSYS.

\subsection{Thermal-hydraulic applications using the ATHLET computer code}

Several uncertainty and sensitivity analyses were performed by GRS using the thermal-hydraulic computer code ATHLET simulating breaks of the primary and secondary side cooling systems of pressurised water reactors. These are

(i) separate effects experiment OMEGA heater rod bundle Test 9,

(ii) integral experiment LSTF-CL-18, 5\% cold leg break, accumulator injection into cold legs,

(iii) PWR 5\% cold leg break, accumulator injection into hot legs (Siemens/ KWU reactor),

(iv) integral experiment LOFT L2-5, $2 \times 100 \%$ cold leg break, accumulator injection into cold legs,

(v) PWR $2 \times 100 \%$ cold leg break, combined ECC injection into cold and hot legs,

(vi) PWR 10\% steam line break,

(vii) PSB-VVER $11 \%$ upper plenum break experiment, UP11-08 (OECD PSB-VVER Test1).

One out of these applications is described in the following section.

\subsection{Application to a German PWR reference reactor, $2 \times 100 \%$ cold leg break}

A double ended cold leg offset shear break design basis accident of a German PWR of 1300 MW electric power is investigated. The fuel rod peak linear heat generation rate is $530 \mathrm{~W} / \mathrm{cm}$. Loss of off-site power at turbine trip is assumed. ECC injection is into cold and hot legs. The accumulator system is specified to initiate coolant injection into the primary system below a pressure of 2.6 MPa. High- and low-pressure ECC injection is available. A single failure is assumed in the broken loop check valve for ECC injection from accumulator, high- and low- pressure system, and one hot leg accumulator is unavailable due to preventive maintenance. These assumptions are considered to be the worst unavailability, agreed between applicants and assessors. 
The uncertainty analysis considered 56 uncertain input parameters. These consist of 37 model parameters, 4 parameters to select different model correlations for heat transfer and friction, 2 for bypass flow cross sections in the reactor vessel, 1 for temperature of accumulator water, 1 for core power, 1 for decay heat, 1 for radial power distribution in the core, 1 for hot channel factor, 5 for gap width (5 burn-up classes), 1 for fuel thermal conductivity, and 2 for convergence criteria. The model parameters comprise critical flow, heat transfer, evaporation, condensation, wall and interfacial shear, form loss, main coolant pump head, and torque.

A total number of 100 calculations were performed using the code ATHLET Mod 1.2, cycle D [8].

\subsection{Maximum clad temperature}

Figure 3 shows at any point of time, at least $95 \%$ of the combined influence of all considered uncertainties on the calculated clad temperatures is below the presented uncertainty limit (one-sided tolerance limit), at a confidence level of at least $95 \%$. For each instant of time, the desired tolerance limits were selected from the 100 calculated code results. A "conservative" calculation result is shown for comparison, applying the best estimate code ATHLET with default values of the models and conservative values for the initial and boundary conditions reactor power, decay heat, gap width of fuel rods between fuel and clad, fuel pellet thermal conductivity, and temperature of accumulator water. All these conservative values were also included in the distributions of the input parameters for the uncertainty analysis. The maximum clad temperature of the conservative calculation does not bound the $95 \% / 95 \%$ one-sided tolerance limits of the uncertainty analysis over the whole transient time, for example, after 75 seconds. The regulatory acceptance criterion for peak clad temperature is $1200^{\circ} \mathrm{C}$.

The "conservative" calculation is representative for the use of best estimate computer codes plus conservative initial and boundary conditions. Such an evaluation is possible in the licensing procedure of several countries, but not in the USA. The uncertainty of code models is not taken into account. The selection of conservative initial and boundary conditions will bound these model uncertainties. That is obviously not the case for the whole transient in the present example. An uncertainty analysis quantifies uncertain initial and boundary conditions as well as model uncertainties. The peak clad temperatures, however, are bounded due to cumulating conservative values of the highly sensitive parameters gap width and pellet thermal conductivity. It is obvious that the results are dependent on the extent of conservatism implemented in the conservative calculations. Therefore, the US Code of Federal Regulation [1] requires that "uncertainties in the analysis method and inputs must be identified and assessed so that the uncertainty in the calculated results can be estimated" when a best-estimate computer code is used for the analysis.

According to the US Code of Federal Regulations, Title 10 , Section 50.46, the conservative method requires conser-

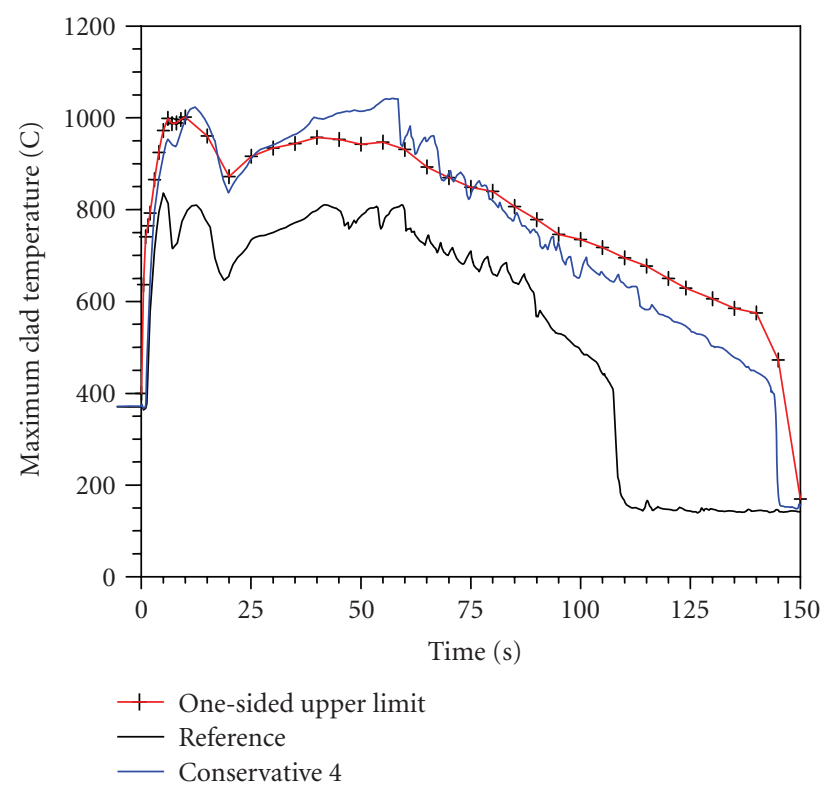

Figure 3: Calculated one-sided 95\%/95\% uncertainty limit and best estimate reference calculation compared with a "conservative" calculation of rod clad temperature for a reference reactor during a postulated double ended offset shear cold leg break.

vative models to be applied in conformity with the required and acceptable features listed in Appendix K, "ECCS Evaluation Models" of the Federal Regulations [1]. This is the main reason why, in the USA, an additional margin to licensing criteria is available by changing from conservative evaluation to best estimate calculations plus uncertainty analysis.

The confidence level 95\% denominates that the 95th percentile is overestimated conservatively by $95 \%$ probability providing a $(95 \%, 95 \%)$ statement. This conservatism is the reason why some experts claim that a coverage of a $(95 \%$, $95 \%$ ) statement by a conservative calculation is not needed. GRS requires coverage unless other suitable methods for comparison and quantification of "conservatisms" are presented. This could be achieved by an additional statistical test proving that the conservative calculation bounds the 95th percentile.

\subsubsection{Sensitivity measures}

Sensitivity measures indicate the influence of the uncertainty in input parameters on calculation results. For example, the Spearman rank correlation coefficient is used as sensitivity measure. The length of the bars indicates the sensitivity of the respective input parameter uncertainty on the first peak clad temperature which occurs during the blowdown phase; see Figure 4 . The sensitivity measure gives the variation of the result in terms of standard deviations when the input uncertainty varies by one standard deviation (if the input uncertainties are independent). Positive sign means that input parameter value and result tend to move in the same direction, that is, an increase of uncertain input parameter value 


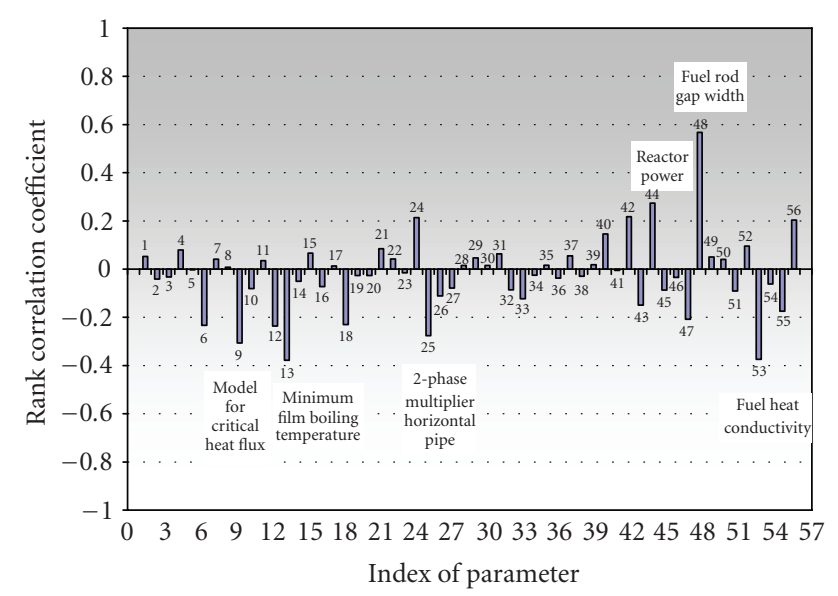

FIgUre 4: Sensitivity measures of the blowdown PCT with respect to the selected 56 uncertain input parameters (rank correlation coefficient) for the reference reactor large break.

tends to increase the clad temperature and vice versa. For negative sign, the input parameter value and the result tend to move in opposite direction, that is, an increase of the parameter value tends to decrease the clad temperature and vice versa.

The most important parameter uncertainties, out of 56 identified potentially important parameters, with respect to the blowdown peak clad temperature uncertainty are

(i) fuel rod gap width for low burn up (positive sign),

(ii) fuel heat conductivity (negative sign),

(iii) minimum film boiling temperature (negative sign),

(iv) model for critical heat flux (negative sign: Biasi correlation causes lower clad temperatures due to a later change from nucleate to transition boiling compared to the Hench-Levy correlation),

(v) reactor initial power (positive sign),

(vi) 2-phase multiplier in horizontal pipe (negative sign: higher resistance of water transport to break location $\Rightarrow$ higher water content in core due to lower break flow $\Rightarrow$ lower clad temperature).

The most important parameters for the peak clad temperature uncertainty during reflood are, according to Figure 5,

(i) fuel heat conductivity (negative sign),

(ii) fuel rod gap width for low burn up (positive sign),

(iii) model for 1-phase convection to steam (positive sign, i.e., Mc Eligot correlation tends to cause higher clad temperatures than Dittus-Boelter II),

(iv) number of droplets (negative sign: number of droplets higher $\Rightarrow$ higher condensation $\Rightarrow$ lower PCT),

(v) steam-droplet cooling (negative sign: higher cooling tends to result in lower PCT).

\subsection{Application to the experiment HDR T31.5 simulating containment behaviour}

The experiment T31.5 on the HDR containment facility simulates a large break of a main coolant pipe, investigating

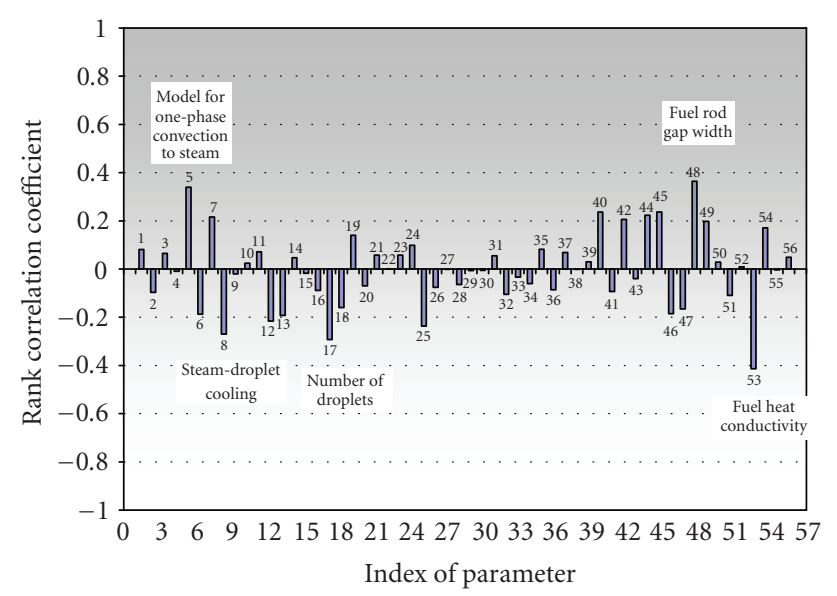

FIGURE 5: Sensitivity measures of the reflood PCT with respect to the selected 56 uncertain input parameters (rank correlation coefficient) for the reference reactor large break.

steam and gas release into the containment according to the low pressure scenario of the German risk study. A short term phase was performed with emphasis on pressure buildup in the containment and the temperature evolution. The hydrogen distribution was measured during a long term phase over 20 hours, when steam and a helium-hydrogen mixture were injected.

A total number of 200 calculations were performed using the code COCOSYS V0.2 [9]. At least 95\% of the combined influence of all considered uncertainties on the calculated pressure at a confidence level of at least $95 \%$ at any point of time is shown in Figure 6. A total of 79 uncertain parameters were included, consisting of model parameters, of the experimental facility, initial and boundary conditions.

Sensitivity measures about the influence of the uncertainty in input parameters on the pressure in the upper part of the HDR containment versus time are presented in Figure 7. We see decreasing and increasing high importance versus time on the maximum pressure. Decreasing influence with time is due to decreasing energy transport with decreasing convection for

(i) free convection, parameter 72, negative sign,

(ii) forced convection, parameter 73, negative sign,

(iii) condensation at wall, parameter 74 , negative sign.

Increasing with time are the following parameters because of decreasing convection:

(i) thickness of liner, parameter 79, negative sign,

(ii) surface of liner, parameter 77 , negative sign,

(iii) heat capacity of concrete structures, parameter 69, negative sign.

\section{CONCLUSIONS}

Two applications of the uncertainty method proposed by GRS are presented. A significant advantage of this methodology is that no a priori reduction in the number of uncertain 


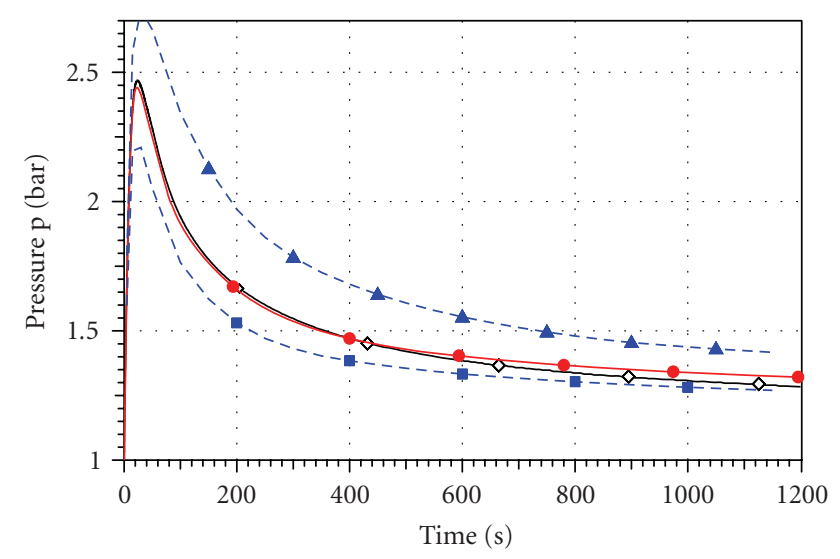

$\diamond$ Experiment CP409

- Reference calculation

- Lower limit

\ Upper limit

Figure 6: 95\%/95\% uncertainty interval, reference calculation and experimental values for pressure in the upper part of the containment versus time.

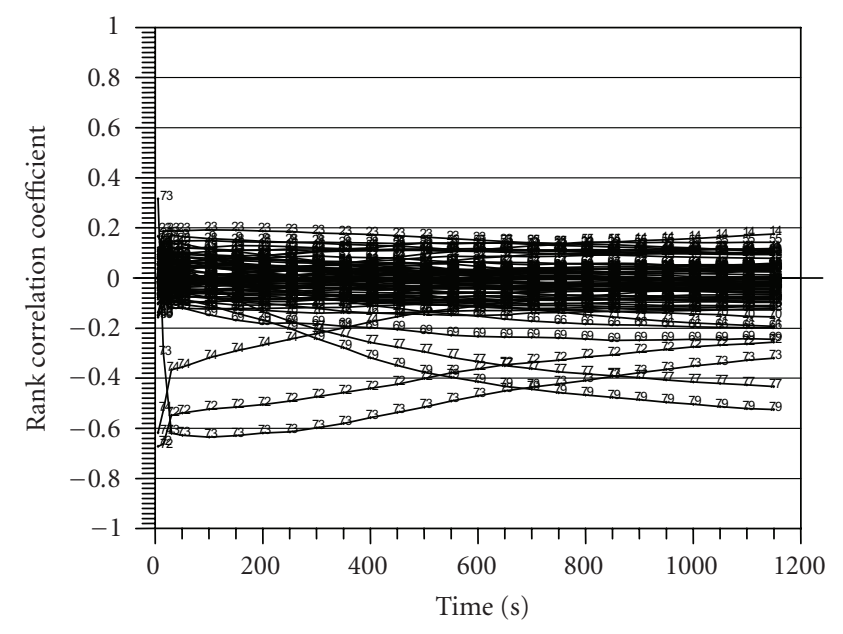

FIgURE 7: Sensitivity measures for pressure in the upper part of the containment versus time.

input parameters by expert judgement or screening calculations is necessary to limit the calculation effort. All potentially important parameters may be included in the uncertainty analysis. The method accounts for the combined influence of all identified input uncertainties on the results. This would be difficult or even impossible to achieve by a priori expert judgement of loss of coolant accidents or transients.

The number of calculations needed is independent of the number of uncertain parameters accounted for in the analysis. It does, however, depend on the requested tolerance limits, that is, the requested probability coverage (percentile) of the combined effect of the quantified uncertainties, and on the requested confidence level of the code results. The tolerance limits can be used for quantitative statements about margins to acceptance criteria.
Another important feature of the method is that it provides sensitivity measures of the influence of the identified input parameter uncertainties on the results. The measures permit an uncertainty importance ranking. This information provides guidance as to where to improve the state of knowledge in order to reduce the output uncertainties most effectively, or where to improve the modelling of the computer code. Different to other known uncertainty methods, the ranking is a result of the analysis and its inputs and not of an a priori expert judgement. Uncertainty statements and sensitivity measures are available simultaneously for all singlevalued (e.g., peak cladding temperature) as well as continuous valued (time dependent) output quantities of interest. The method relies only on actual code calculations without the use of approximations like fitted response surfaces. The method proposed by GRS has been used in different applications by various international institutions including licensing.

A challenge in performing uncertainty analyses is the specification of ranges and probability distributions of input parameters. Investigations are underway to transform data measured in experiments and post test calculations into thermal-hydraulic model parameters with uncertainties. Care must be taken to select suitable experimental and analytical information to specify uncertainty distributions. This is a general experience gained in applying different uncertainty methods.

\section{ACKNOWLEDGMENTS}

This work performed by GRS was funded by the German Federal Ministry for Economy under Contract no. RS 1142. The significant contributions of my colleagues H. Bartalsky, A. Hora, B. Krzykacz-Hausmann, and T. Skorek to this paper are gratefully acknowledged.

\section{REFERENCES}

[1] 10 CFR 50.46, "Acceptance criteria for emergency core cooling systems for light water nuclear power reactors," Appendix K, "ECCS Evaluation Models", to 10 CFR Part 50, Code of Federal Regulations, 1996.

[2] E. Hofer, "Probabilistische Unsicherheitsanalyse von Ergebnissen umfangreicher Rechenmodelle," GRS-A-2002, January 1993.

[3] S. S. Wilks, "Determination of sample sizes for setting tolerance limits," Annals of Mathematical Statistics, vol. 12, no. 1, pp. 9196, 1941.

[4] S. S. Wilks, "Statistical prediction with special reference to the problem of tolerance limits," Annals of Mathematical Statistics, vol. 13, no. 4, pp. 400-409, 1942.

[5] B. E. Boyack, I. Catton, R. B. Duffey, et al., "Quantifying reactor safety margins," Nuclear Engineering and Design, vol. 119, no. 1, pp. 1-15, 1990.

[6] Bemuse Phase III Report, "Uncertainty and sensitivity analysis of the LOFT L2-5 test," Tech. Rep. NEA/CSNI/R(2007)4, Nuclear Energy Agency, Issy-les-Moulineaux, France, October 2007.

[7] M. Kloos and E. Hofer, SUSA - PC, a personal computer version of the program system for uncertainty and sensitivity analysis of results from computer models, version 3.2, user's guide and 
tutorial, Gesellschaft für Anlagen- und Reaktorsicherheit, Garching, Germany, August 1999.

[8] G. Lerchl and H. Austregesilo, ATHLET Mod 1.2 User's Manual. GRS-P-1 / Vol.1, Rev. 2a, November 2000.

[9] W. Klein-Heßling, COCOSYS V0.2, User Manual. GRS-P-3/1. 

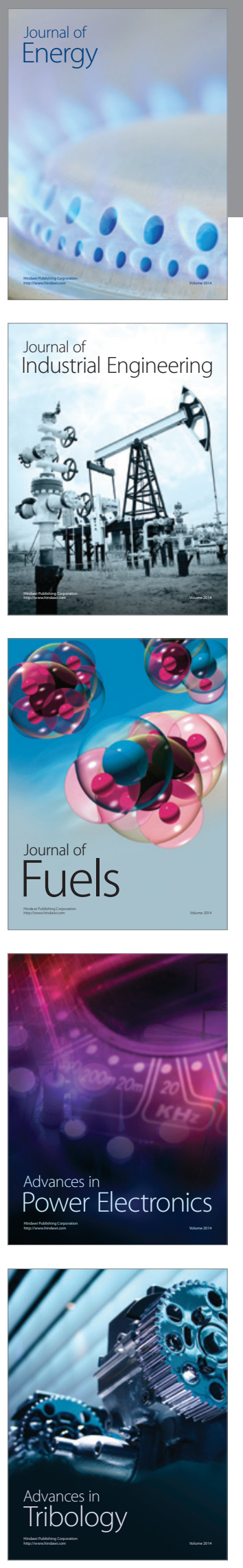
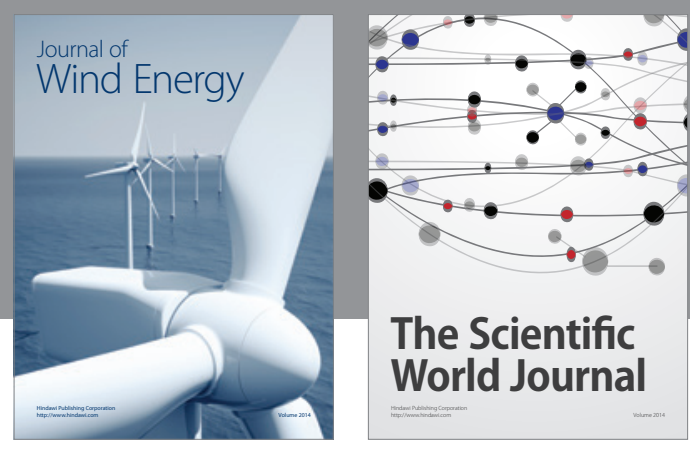

The Scientific World Journal

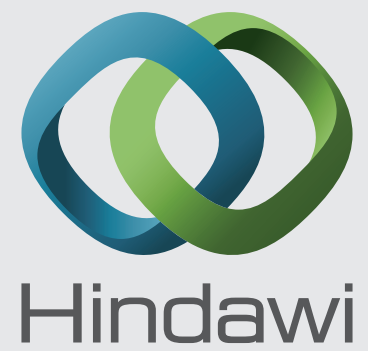

Submit your manuscripts at http://www.hindawi.com
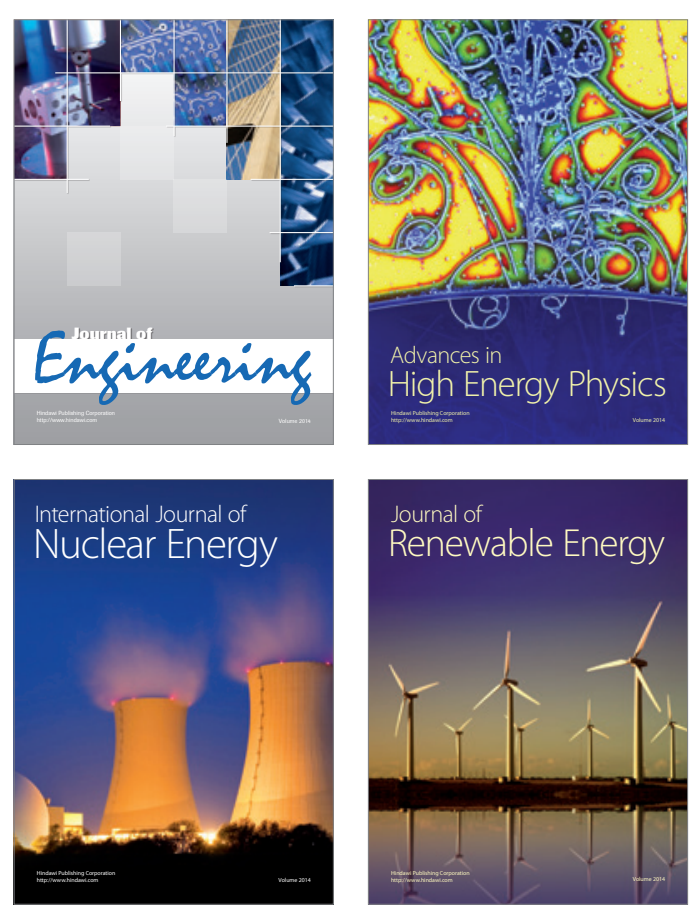

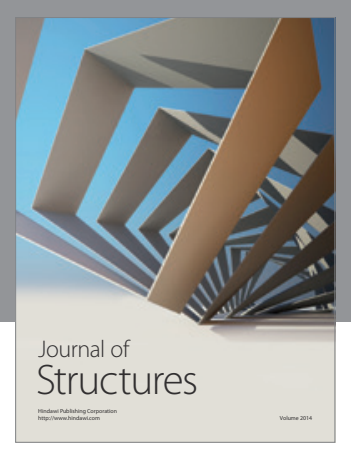

Rotating
Mechinery
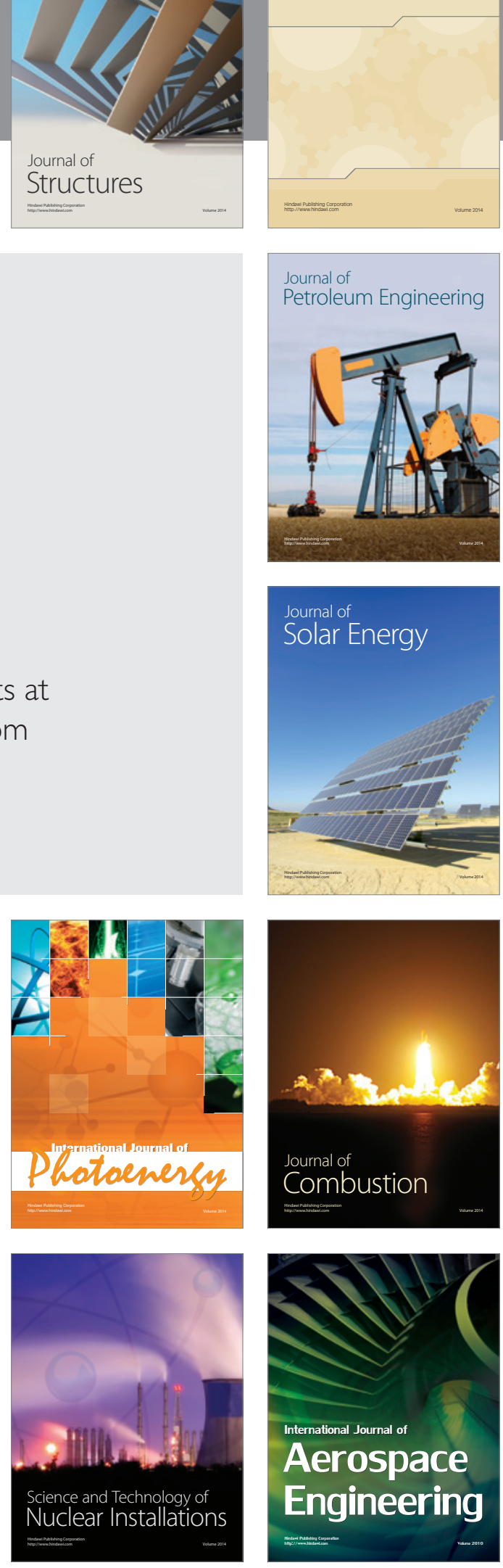